\title{
Hadronic Physics in Geant4: Improvements and Status for LHC Start
}

\author{
Vladimir Ivanchenko ${ }^{1}$, John Apostolakis, Gunter Folger, Vladimir Grichine, \\ Alexander Howard, Anton Ivantchenko, Mikhail Kossov, Alberto Ribon, Vladimir \\ Uzhinsky \\ CERN \\ CH-1211 Geneve 23, Switzerland \\ E-mail: Vladimir.Ivantchenko@cern.ch, John.Apostolakis@cern.ch, Gunter.Folger@cern.ch
}

\section{Sunanda Benerjee, Julia Yarba}

Fermilab,

Box 500, Batavia, Illinois 60510, USA

E-mail:sunanda@fnal.gov, Julia.Yarba@,cern.ch

Miguel Cortes, Jose Manuel Quesada

University of Seville

E-41080 Sevilla, Spain

E-mail: miancortes@us.es,quesaeda@us.es

\section{Tatsumi Koi, Dennis Wright}

SLAC

Stanford, CA 94309, USA

E-mail: Tatsumi.Koi@slac.stanford.edu, Dennis.Wright@slac.stanford.edu

\begin{abstract}
An overview of recent developments in Geant4 hadronic modelling is provided with a focus on the start of the LHC experiments. Improvements in low-energy and high energy models were introduced. Many of these developments were directed toward increasing the precision of simulated hadronic showers for LHC detectors. Theoretical arguments were used as well as tuning to thin target experiment data. The testing suite for the Geant 4 hadronic models was extended. Selected validation results are presented. The variants of Geant 4 physics model configurations (Physics Lists) for LHC experiments are discussed.
\end{abstract}

XII Advanced Computing and Analysis Techniques in Physics Research

Erice, Italy

3-7 November, 2008

Speaker 


\section{Introduction}

The Geant4 toolkit $[1,2]$ has been used for many years for the Monte Carlo simulation of LHC experiments. Hadronic physics packages [3] are responsible for the simulation of detector response to hadrons, in particular hadronic showers in calorimeters. For any hadron energy the response depends of simulation of both high energy and low energy processes, including the low-energy neutron transport. The configuration of Geant4 hadronic models is provided in term of Physics Lists [4]. Since release 8.3 (May 2007), the QGSP_BERT Physics List was adopted for simulation production both by ATLAS and CMS.

In this work we highlight recent improvements of Geant4 hadronic physics models. The efforts were concentrated on providing better agreement of simulation results with the data of thin target experiments and the LHC test-beam data. To achieve this goal the hadronic testing suite [5] has been significantly extended [6,7]. It covers an energy range of primary hadrons from $20 \mathrm{MeV}$ to $400 \mathrm{GeV}$ and allows validation of double differential cross sections for neutron, proton, charged pion production. As a result, the precision of the QGSP_BERT simulation is increased and alternative Physics Lists based on Fritiof string model are provided.

\section{String models}

In Physics Lists used for LHC, high energy hadron-nuclear interactions are simulated using the quark-gluon string model (QGS), which is valid above $12 \mathrm{GeV}$. Initially this model provided hadronic shower in a calorimeter [3] which were shorter and narrower than expected. A refinement of the cross section for the quasi-elastic scattering of high energy primary pions allows the longitudinal shower shape to be nearly reproduced [8]. Recently the quasi-elastic model was updated by inclusion of Fermi-motion for target nucleons providing a smooth spectrum of recoil nucleons.

The sampling of low-energy secondary particles in QGSP-type Physics Lists is performed using the Pre-Compound model [1,3]. The simulated high energy part of the spectrum of produced hadrons (Fig.1) is close to the data [9] but the low energy part is absent. This problem is resolved by introduction of the Binary cascade [10] or CHIPS model [11] for sampling the rescattering of the low-energy component of nuclear fragmentation (QGS_BIN or QGSC).

The main problems of Physics Lists based on QGS model is in the simulation of diffractive processes and in the validity gap between cascade and string models around $10 \mathrm{GeV}$. To address these issues an alternative string model (FTF) was developed, which is based on the classical Fritiof approach [12]. The FTF model allows simulation of the quasi-elastic scattering and the diffraction processes. The following recent modifications have been introduced [13]:

- refinement of the quasi-elastic scattering cross section and the Fermi momentum distribution of nucleons,

- tuning of the diffractive dissociation cross section to experimental data,

- implementation of formation time for secondary particles, and

- employing the Binary cascade for sampling of the cascade of slow particles. 


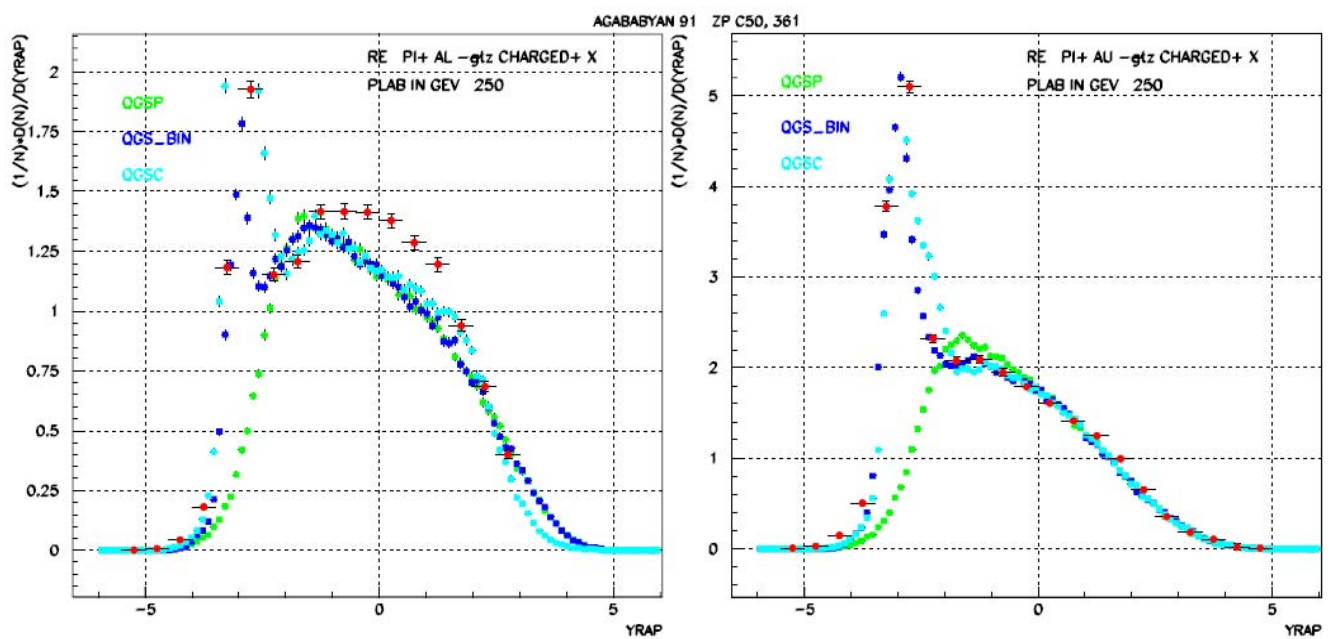

Fig.1 Invariant cross section as a function of rapidity of charged secondary hadrons produced by collision of $250 \mathrm{GeV} \pi^{+}$with Aluminum (left) and Gold (right) targets: red points - data [9], green dots QGSP, blue dots - QGS_BIN, blue light dots - QGSC.

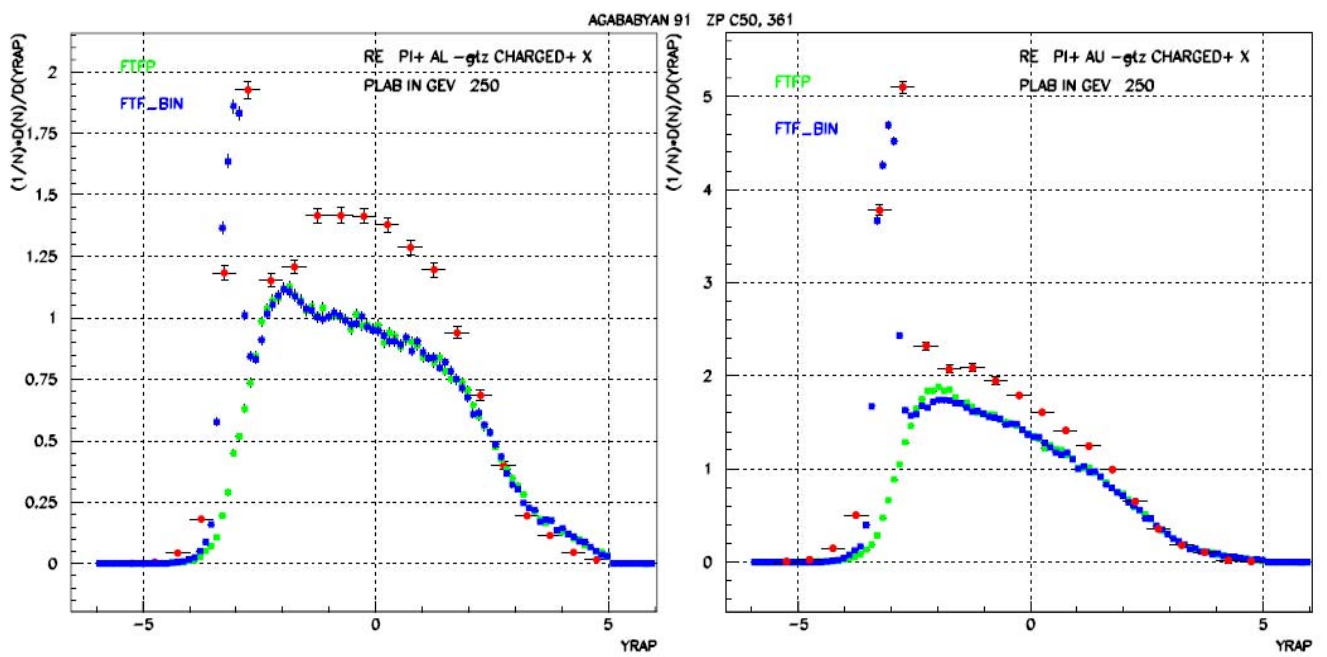

Fig.2 Invariant cross section as a function of rapidity of charged secondary hadrons produced by collision of $250 \mathrm{GeV} \pi^{+}$with Aluminum (left) and Gold (right) targets: red points - data [9], green dots FTFP, blue dots-FTF_BIN.

FTF model predictions (Fig.2) for the high rapidity $(>2)$ distributions are of the same precision as QGS, but for the moderate rapidity FTF underestimates number of secondaries. FTF_BIN is needed to describe low energy particles re-scattering. The main advantage of the updated FTF model is its ability of simulate down to $\sim 5 \mathrm{GeV}$, because of the natural sampling of the diffractive processes dominating the moderate energy region. This is confirmed by comparison with data [14] on pion production (Fig.3). FTF with the Binary cascade (FTFB) and Bertini cascade (BERT) are closer to the data than the QGS-based models. 


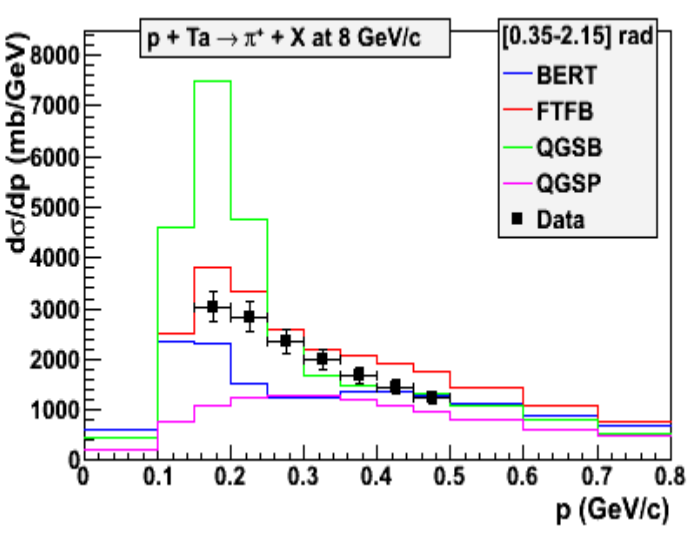

Fig. 3 Inclusive cross section of $\pi^{+}$production by proton beam on Tantalum target: points data [14], histograms - Geant4 models.

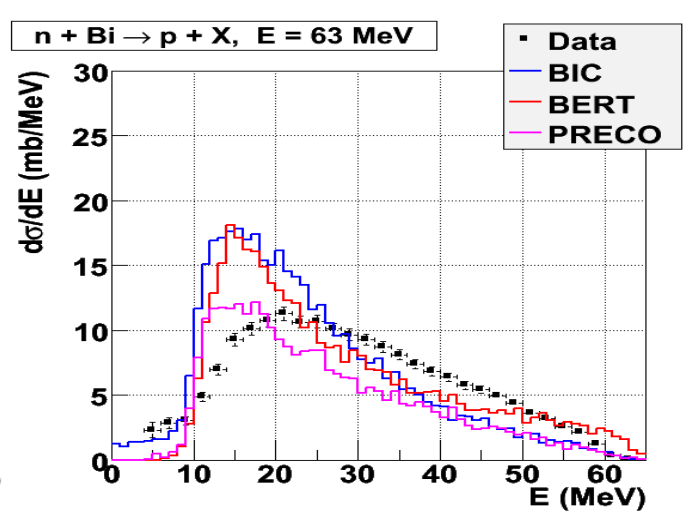

Fig.4 Proton production by $63 \mathrm{MeV}$ neutron beam on Bismuth target: points - data [15], histograms - Geant4 models.

\section{Cascade and low energy models}

Recently it was understood that the Bertini cascade model is needed for the LHC simulation $[4,8]$. Because hadronic calorimeter responses are mainly determined by the production of $\pi^{0}$ and protons the model was validated against various thin target data for $\pi^{ \pm}$and proton production. The following improvements were recently introduced:

- correct normalization of the quasi-elastic cross section (Fig.3),

- improved partial cross sections shape, and

- Coulomb barrier is added in the pre-compound and cascade phases (Fig.4).

A review of the native Geant4 Pre-Compound and de-excitation models was also carried out [16]. An important characteristic of simulation in the cascade energy range is the description of inclusive neutron production, which is reproduced by Geant4 cascades (Fig.5).
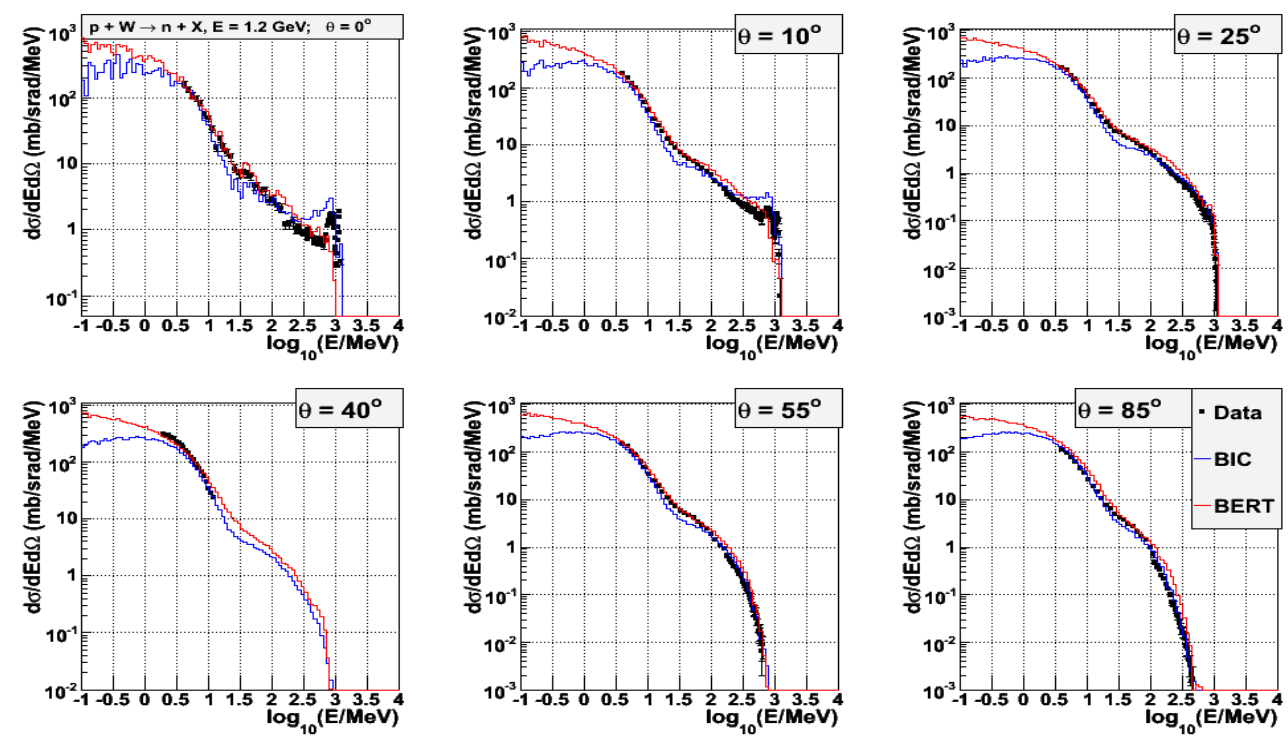

Fig. 5 Inclusive double differential cross section of the neutron production by the $1.2 \mathrm{GeV}$ proton beam on the Tungsten target: points - data [17], histograms - Geant4 cascade models. 


\section{Conclusions}

Described developments of the Geant 4 hadronic models provide better agreement between simulation predictions and the data from thin target experiments with the QGSP_BERT Physics List, which is default for most LHC detectors. Refinement of the FTF model makes it possible to use FTF-based Physics Lists as an alternative. Updated models are available with Geant4 release 9.2, which can be considered for the simulation of first collision experiments at LHC.

\section{References}

[1] S. Agostinelli et al., Geant4: A simulation toolkit, Nucl. Instrum. Meth. A 506 (2003) 250.

[2] J. Allison et al., Geant4 developments and applications, IEEE Trans. Nucl. Sci. 53 (2006) 270.

[3] D. H. Wright et al., Low and high energy modelling in Geant4, AIP Conf. Proc. 896 (2007) 11.

[4] A. Ribon et al., Geant4 Physics Lists: improvements and status, in proceedings of IEEE NSS conference, Dresden, Germany, 19 - 25 October 2008, NSS N02-89.

[5] V. N. Ivanchenko and A. Ivantchenko, Testing suite for validation of Geant4 hadronic generators, J. Phys.Conf. Ser.119 (2008) 032026.

[6] G. Folger et al., Validation of high energy string models in Geant4 , in proceedings of IEEE NSS conference, Dresden, Germany, 19 - 25 October 2008, NSS N37-4.

[7] S. Benerjee et al., Validation of Geant4 hadronic physics models at intermediate energies, in proceedings of IEEE NSS conference, Dresden, Germany, 19 - 25 October 2008, NSS N37-5.

[8] S. Piperov, Calorimeter simulation with hadrons in CMS, in proceedings of IEEE NSS conference, Dresden, Germany, 19 - 25 October 2008, NSS N37-8.

[9] N. M. Agababyan et al., Rapidity and transverse momentum structure in $\pi^{+}$and $\mathrm{K}^{+}$collisions with Al and Au nuclei at $250 \mathrm{GeV/c}$, Z. Phys C 50 (1991) 361.

[10] G. Folger, V. N. Ivanchenko, H. P. Wellisch, The Binary cascade, Eur. Phys. Jour. A 21 (2004) 407

[11] L. V. Degtyarenko, M. V. Kossov, H. P. Wellisch, Chiral invariant space generator, Eur. Phys. J. A 8 (2000) 217; 9 (2000) 411; 9 (2000) 421.

[12] B.Anderson et al., A model for low-pT hadronic reactions with generalizations to hadron-nucleus and nucleus-nucleus collisions, Nucl. Phys. 281 (1987) 289.

[13] V. Uzhinsky, Fritiof model in Geant4, in proceedings of IEEE NSS conference, Dresden, Germany, 19 - 25 October 2008, NSS N37-7.

[14] M. G. Catanesi et al., Large-angle production of charged pions with 3 - $12.9 \mathrm{GeV} / \mathrm{c}$ incident protons on nuclear targets, Phys. Rev. C 77 (2008) 055207.

[15] E. Raeymackers et al., Light charged particle emission in fast neutron (25 -65 MeV) induced reactions on Bi-209, Nucl. Phys. A 726 (2003) 210.

[16] J. M. Quesada et al., Improvements of preequilibrium and evaporation models in Geant4, in proceedings of IEEE NSS conference, Dresden, Germany, 19 - 25 October 2008, NSS N02-97.

[17] S. Leray et al., Spallation neutron production by 0.8, 1.2, and $1.6 \mathrm{GeV}$ protons on various targets, Phys. Rev. C 65 (2002) 044621. 